\title{
Charm physics at ATLAS
}

\author{
Semen Turchikhin ${ }^{1, *}$, on behalf of the ATLAS Collaboration** \\ ${ }^{1}$ Joint Institute for Nuclear Research, Dubna, Russia
}

\begin{abstract}
This proceeding contribution presents the recent highlights of charm physics programme of the ATLAS experiment at the Large Hadron Collider. These include measurements of charmonia production both in $p p$ and nuclear collisions, charmonium pair production, studies of exotic states and $B_{c}$ mesons. Most of the shown results are obtained with the data collected during the LHC Run-1.
\end{abstract}

\section{Introduction}

The ATLAS experiment [1] is a general purpose detector at LHC designed for a wide range of particle physics studies. Heavy flavour studies is an essential part of its physics programme. Coverage of the central rapidity region and very large available statistics of $p p$ collision data makes ATLAS very competitive in certain statistically limited studies.

Studies of charm physics at ATLAS are mostly limited to those involving the $J / \psi$ meson decaying into a muon pair, due to limitations imposed by the ATLAS trigger. A number of such studies is presented below. These are measurements of charmonium state production in $p p$ as well as in $\mathrm{PbPb}$ and $p \mathrm{~Pb}$ collisions, of double charmonia production, studies of exotics in charm sector, of the $B_{c}$ meson family.

\section{Charmonia production in $p p$ collisions}

In hadronic collisions, charmonium states can be produced either directly by short-lived QCD sources ("prompt" production), or in the decay chains of beauty hadrons ("non-prompt" production). Fixed-Order with Next-to-Leading-Log (FONLL) calculations within the framework of perturbative QCD were shown to be quite successful in describing the non-prompt charmonia production, while for the prompt production non-relativistic QCD (NRQCD) calculations represent the most common theoretical approach. The latter include colour-octet contributions and introduce a number of long-distance matrix elements - phenomenological parameters determined from fits to earlier collider data, that allow to achieve a satisfactory description of the cross-sections and differential spectra.

ATLAS has performed a precise measurement [2] of $J / \psi$ and $\psi(2 S)$ production at centreof-mass energies $\sqrt{s}=7$ and $8 \mathrm{TeV}$ using the statistics of $2.1 \mathrm{fb}^{-1}$ and $11.4 \mathrm{fb}^{-1}$, respectively. Prompt and non-prompt production contributions are determined with a two-dimensional mass and pseudo-proper lifetime fit. The measurement is performed in the rapidity region $|y|<2.0$ and covers the $p_{\mathrm{T}}$ range between 8 and $110 \mathrm{GeV}$.

\footnotetext{
*e-mail: Semen.Turchikhin@cern.ch

** Copyright 2019 CERN for the benefit of the ATLAS Collaboration. CC-BY-4.0 license.
} 
Figure 1 shows the doubly differential $p_{\mathrm{T}}$ and rapidity spectra of prompt and non-prompt $J / \psi$ compared to the corresponding theoretical predictions. In addition, $\psi(2 S)$ spectra, ratio of spectra between the two charmonia states and between the two centre-of-mass energies, fractions of non-prompt production for $J / \psi$ and $\psi(2 \mathrm{~S})$ are measured. These observables are of interest for understanding of the underlying production mechanisms. The predictions from the NRQCD model for the prompt production mechanism are found to be in a good agreement with the data. For the non-prompt production, the FONLL calculations reproduce the data reasonably well, with a slight overestimation of the differential cross-sections near the upper bound of the $p_{\mathrm{T}}$ range reached by the measurement. The ATLAS results extend the range of existing measurements to the higher $p_{\mathrm{T}}$ range, and are consistent with previous measurements made by ATLAS and other LHC experiments in overlapping phase-space regions.
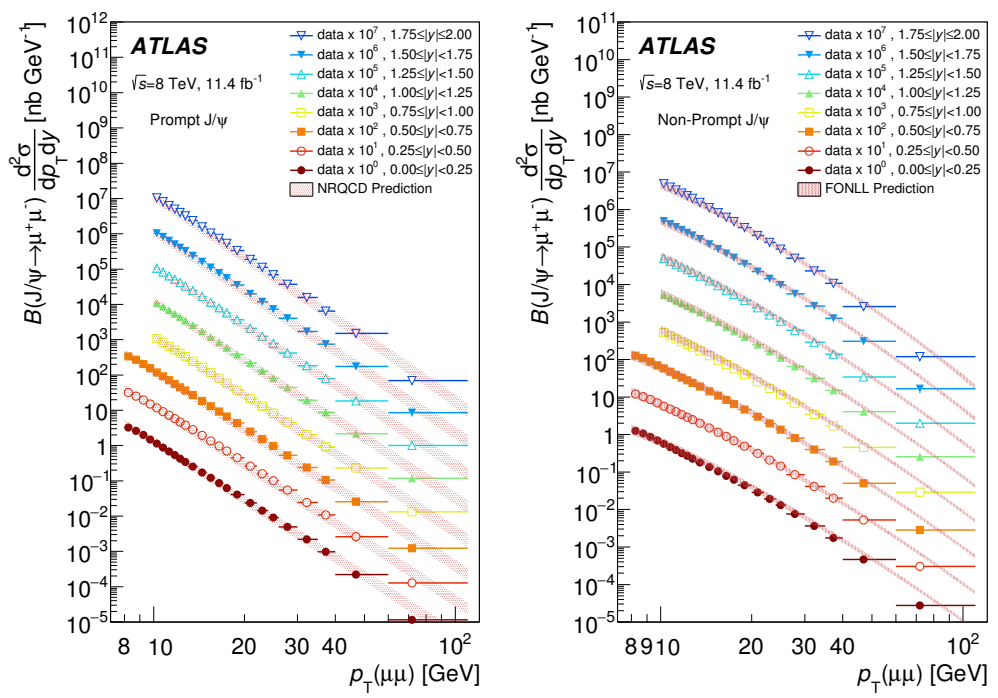

Figure 1. The differential cross-section times dimuon branching fraction for (left) prompt and (right) non-prompt $J / \psi$ production at $\sqrt{s}=8 \mathrm{TeV}$ as a function of $p_{\mathrm{T}}$ in slices of rapidity. Figures are taken from Ref. [2].

With early Run-2 data ATLAS has performed a measurement of the fraction of $J / \psi$ produced through the non-prompt mechanism at $\sqrt{s}=13 \mathrm{TeV}$. It is shown in Fig. 2, along with the comparison to earlier measurements in the same or comparable rapidity intervals. The shape is found to be similar to that at $\sqrt{s}=7 \mathrm{TeV}$, contrary to the significant difference observed between the $\sqrt{s}=7 \mathrm{TeV}$ measurement and those at lower energies.

\section{Charmonia production in nuclear collisions}

Quarkonia production in ultra-relativistic heavy ion collisions is a valuable probe of the deconfined quark-gluon plasma. A possible suppression of the production can provide information about the temperature and degree of the deconfinement. Enhancement of the production could also appear when e.g. quarkonium is formed due to recombination of $c \bar{c}$ pair from the medium. Studying the non-prompt quarkonia production is also important as it allows to probe the $b$ quark propagation through the medium. The $b$ quark production possibly suffers from different suppression mechanisms (energy loss by collisional or radiative processes) than the $c \bar{c}$ production (colour screening in the medium). 

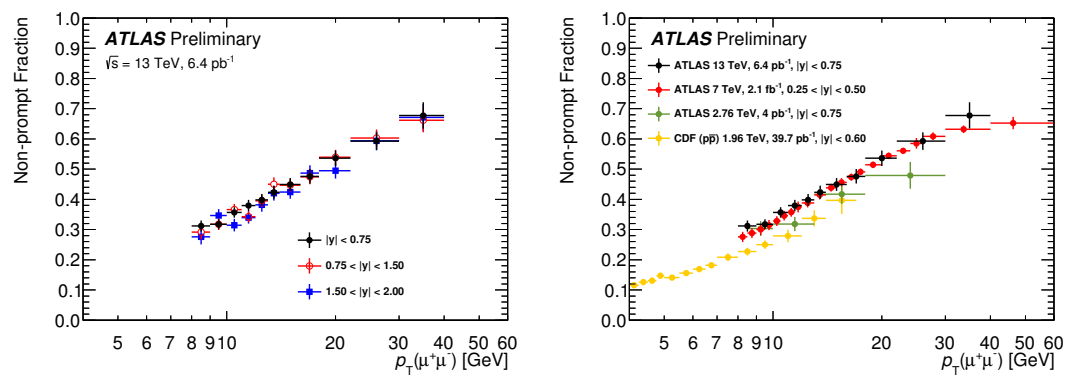

Figure 2. (Left) non-prompt $J / \psi$ production fraction as a function of $p_{\mathrm{T}}$ and in three intervals of rapidity and (right) comparison of the measurement in the most central rapidity region $|y|<0.75$ to previous measurements by ATLAS and CDF at smaller energies. Figures are taken from Ref. [3].

The ATLAS measurement [4] of $J / \psi$ and $\psi(2 \mathrm{~S})$ production in $\mathrm{PbPb}$ collisions at $\sqrt{s_{\mathrm{NN}}}=$ $5.02 \mathrm{TeV}$ and in $p p$ collisions at $\sqrt{s}=5.02 \mathrm{TeV}$ is done using data collected in 2015 corresponding to integrated luminosities of $0.42 \mathrm{nb}^{-1}$ and $25 \mathrm{pb}^{-1}$, respectively.

Figure 3 shows the nuclear modification factor $R_{\mathrm{AA}}$ for prompt and non-prompt $J / \psi$ production $^{1}$. Strong suppression of prompt and non-prompt charmonia is observed in $\mathrm{PbPb}$ data. The maximum suppression of prompt and non-prompt $J / \psi$ production by factor $4-5$ is observed for the most central collisions. While the production of prompt $J / \psi$ mesons is suppressed slightly more in the mid-centrality region, the two classes of meson production have essentially the same pattern. This is not quite expected because the two cases are believed to have rather different physical origins: the non-prompt production should be dominated by propagation of $b$ quark that extends far outside the deconfined medium, whereas the prompt production happens predominantly within the medium.

The double ratio of $\psi(2 \mathrm{~S})$ to $J / \psi$ production $\rho_{\mathrm{PbPb}}^{\psi(2 \mathrm{~S}) / J / \psi}=$ $\left(N_{\psi(2 \mathrm{~S})} / N_{J / \psi}\right)_{\mathrm{PbPb}} /\left(N_{\psi(2 \mathrm{~S})} / N_{J / \psi}\right)_{p p}$ is shown in Fig. 4. The ratio is consistent with unity for non-prompt mesons originating from $b$ quarks loosing energy in the medium. For prompt mesons it is different from unity, thus indicating the enhanced suppression of prompt $\psi(2 \mathrm{~S})$ relative to $J / \psi$. This observation is consistent with the interpretation that the $J / \psi$, being a tightly bound quarkonium system, survives the temperature of the hot and dense medium with a higher probability than the more loosely bound $\psi(2 \mathrm{~S})$ state.

Study of heavy quarkonium production in $p \mathrm{~Pb}$ collisions, where a formation of a large region of hot and deconfined matter is not expected, allows to probe Cold Nuclear Matter (CNM) effects. The CNM quarkonium suppression effects can appear either in the initial state (modifications of the nuclear parton distribution functions, parton saturation in the incident nucleus, or parton energy loss through interactions with the nuclear medium), or in final state (absorption of the pairs of heavy quarks).

ATLAS has performed a measurement of the modification of the $J / \psi, \psi(2 S)$ and $\Upsilon(n S)$ $(n=1,2,3)$ states production in $p \mathrm{~Pb}$ collisions with respect to that in $p p$ collisions using the $p \mathrm{~Pb}$ and $p p$ datasets both collected at a centre-of-mass energy $5.02 \mathrm{TeV}$ and corresponding to integrated luminosities of $28 \mathrm{nb}^{-1}$ and $25 \mathrm{pb}^{-1}$, respectively.

The nuclear modification factor $R_{p \mathrm{~Pb}}=1 / 208 \cdot \sigma_{p \mathrm{~Pb}} / \sigma_{p p}$ for $\Upsilon(1 \mathrm{~S})$ and prompt $J / \psi$ production as a function of $p_{\mathrm{T}}$ is shown in Fig. 5. The $R_{p \mathrm{~Pb}}$ is consistent with unity for $J / \psi$

\footnotetext{
${ }^{1}$ The nuclear modification factor is defined as $R_{\mathrm{AA}}=N_{\mathrm{AA}} /\left(\left\langle T_{\mathrm{AA}}\right\rangle \times \sigma_{p} p\right)$, where $N_{\mathrm{AA}}$ is the per-event $J / \psi$ yield in the nuclear collisions, $\sigma_{p p}$ is the production cross-section in $p p$ collisions at the same energy, and $\left\langle T_{\mathrm{AA}}\right\rangle$ is the mean nuclear thickness function.
} 

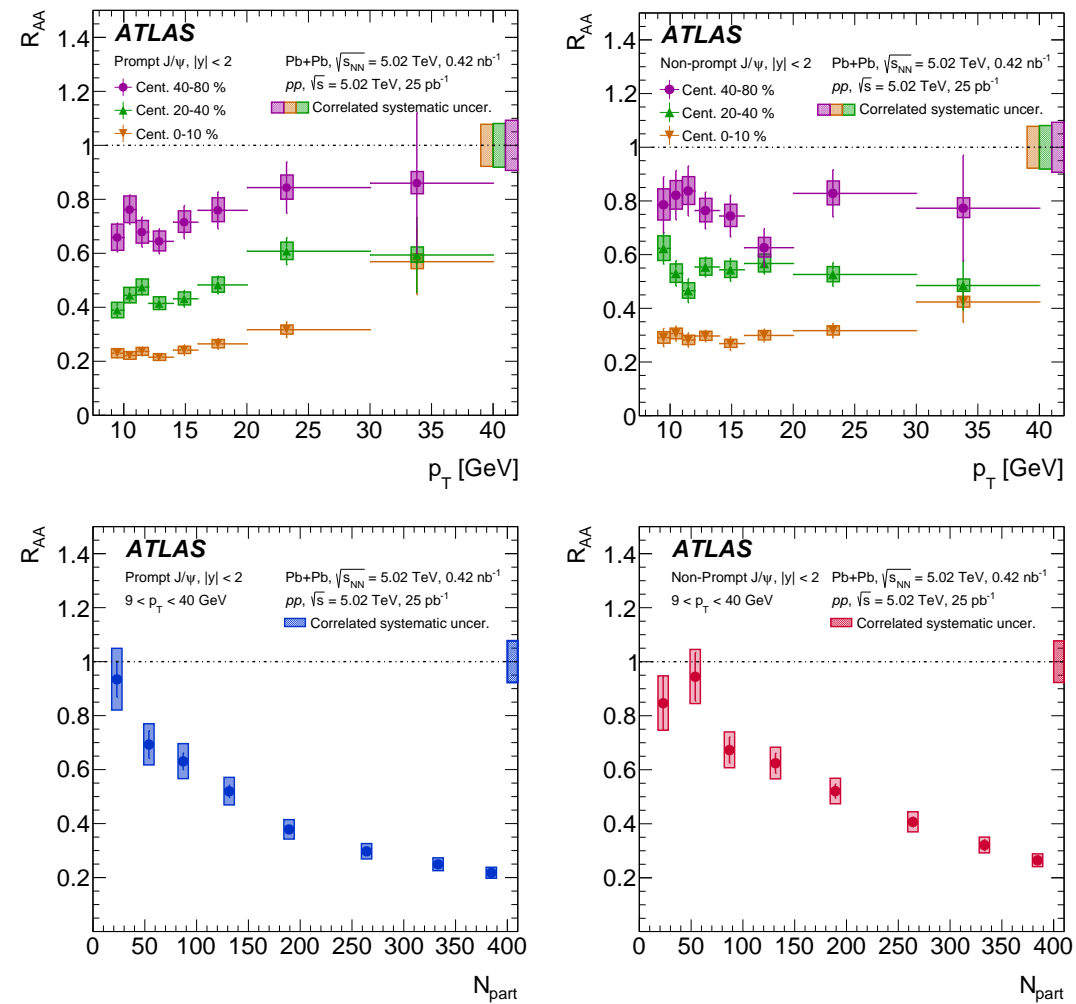

Figure 3. The nuclear modification factor as a function (top) of $p_{\mathrm{T}}$ in different centrality bins and (bottom) of the number of nucleons participating in a collision, $N_{\text {part }}$, for the (left) prompt and (right) non-prompt $J / \psi$. Figures are taken from Ref. [4].
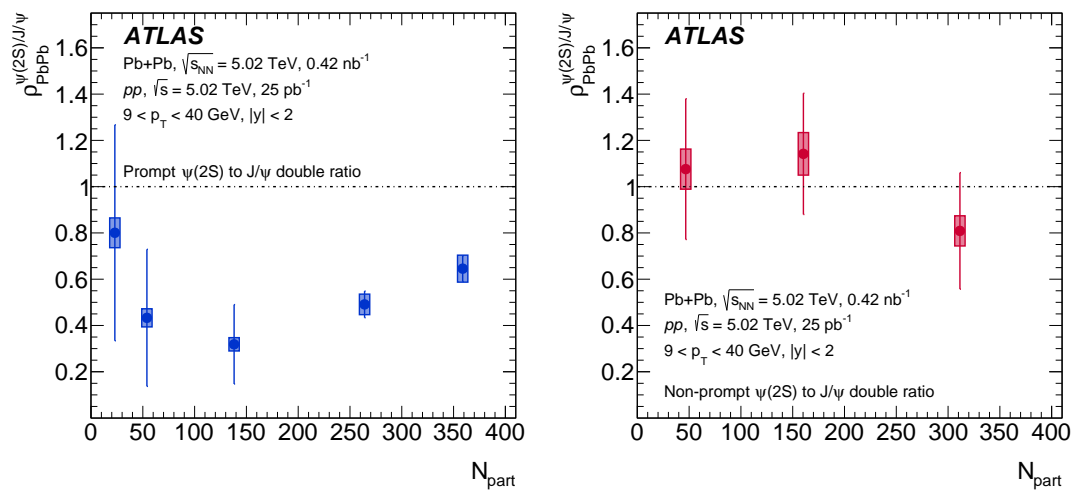

Figure 4. $\psi(2 \mathrm{~S})$ to $J / \psi$ double ratio, as a function of the number of nucleons participating in a collision, $N_{\text {part }}$, for the (left) prompt and (right) non-prompt meson production. Figures are taken from Ref. [4]. 
while for $\Upsilon(1 \mathrm{~S})$ a suppression at low $p_{\mathrm{T}}$ appears. The latter suggests that the nuclear parton distribution functions are modified relative to those of the nucleon.
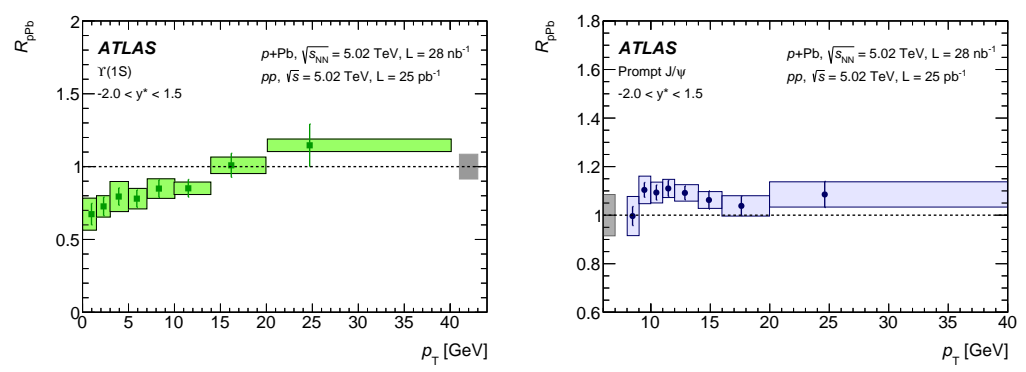

Figure 5. Nuclear modification factor as a function of $p_{\mathrm{T}}$ for (left) $\Upsilon(1 \mathrm{~S})$ and (right) prompt $J / \psi$ production. Figures are taken from Ref. [5].

Figure 6 shows the double ratios $\rho_{p \mathrm{~Pb}}^{n \mathrm{~S} / 1 \mathrm{~S}}$ as a function of the $p \mathrm{~Pb}$ collision centrality. A suppression of higher states is observed with a significance of $1 \sigma$ for $\psi(2 S)$ and $2 \sigma$ for $\Upsilon(2,3 S)$. The effect increases with centrality and suggests strong CNM effects for excited quarkonia.
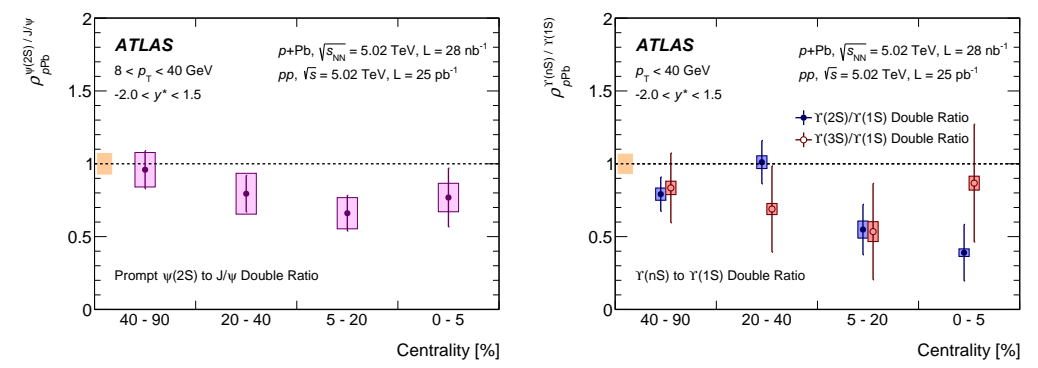

Figure 6. The double ratio $\rho_{p \mathrm{~Pb}}^{n \mathrm{~S} / 1 \mathrm{~S}}$ for (left) prompt charmonium and (right) bottomonium as a function of the collision centrality in the kinematic ranges indicated on the plots. The vertical size of the leftmost box corresponds to the total uncertainty of the $p p$ reference. Figures are taken from Ref. [5].

\section{$4 \mathrm{~J} / \psi$ pair production}

A $J / \psi$ pair can be produced in $p p$ collisions either by single parton scattering (SPS) or by double parton scattering (DPS) via two independent parton-parton interactions. The study of these processes provides a better understanding of higher order and non-perturbative QCD.

A simplified ansatz for defining the DPS cross-section in terms of the production crosssections of the two final states and an effective cross-section, $\sigma_{\text {eff }}$, renders for di- $J / \psi$ production as

$$
\sigma_{\mathrm{eff}}=\frac{1}{2} \frac{\sigma_{J / \psi}^{2}}{\sigma_{\mathrm{DPS}}^{J / \psi, J / \psi}}=\frac{1}{2} \frac{\sigma_{J / \psi}^{2}}{f_{\mathrm{DPS}} \times \sigma_{J / \psi J} / \psi} .
$$

The $\sigma_{\text {eff }}$ is related to the spatial separation between partons inside the proton and is assumed to be independent of energy and of parton interaction process to a first-order approximation in perturbative QCD predictions. 
ATLAS measurement of prompt $J / \psi$ pair production cross-section [6] uses the $p p$ collision data collected at $\sqrt{s}=8 \mathrm{TeV}$ corresponding to an integrated luminosity of $11.4 \mathrm{fb}^{-1}$.

Using $J / \psi$ reconstructed from two independent events in data as a template for DPS, the fraction of prompt-prompt $J / \psi$ pairs produced via DPS is measured to be $f_{\mathrm{DPS}}=(9.2 \pm$ 2.1 (stat.) \pm 0.5 (syst.)) $\%$. The effective cross-section is measured to be $\sigma_{\text {eff }}=6.3 \pm 1.6$ (stat.) \pm 1.0 (syst.) $\pm 0.1(\mathrm{BF}) \pm 0.1$ (lumi.) $\mathrm{mb}$, where the $\mathrm{BF}$ uncertainty corresponds to the uncertainty of $\mathcal{B}\left(J / \psi \rightarrow \mu^{+} \mu^{-}\right)$. Similarly to results of other experiments, this value extracted using di-quarkonium states is lower than those measured for other final states.

Figure 7 shows the differential $J / \psi$ pair production cross-section and the estimated DPS contribution. The data is in a reasonable agreement with the predictions made in leading order QCD for the DPS and next-to-leading order colour singlet non-relativistic QCD calculations without loops (NLO*) for the SPS contribution.
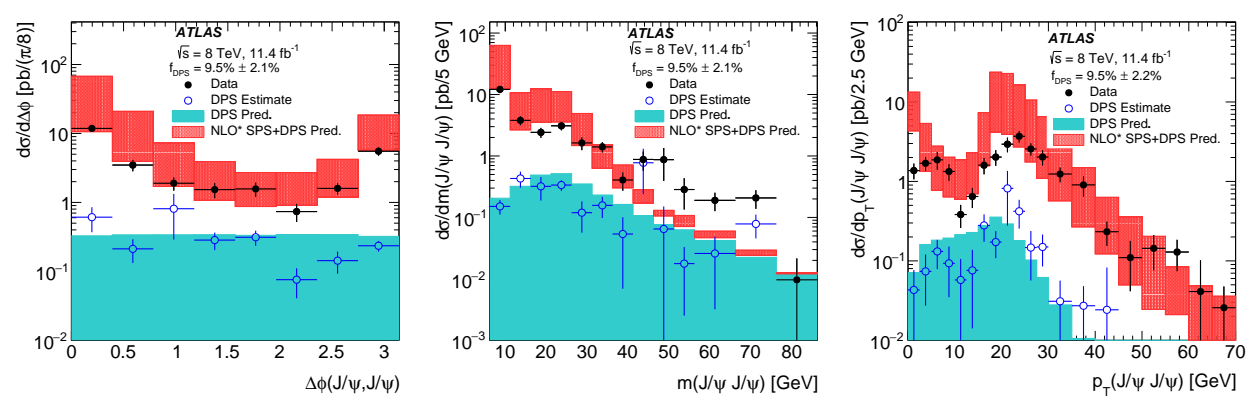

Figure 7. The DPS and the total differential $J / \psi$ pair production cross-section as a function of (left) the azimuthal angle between the two $J / \psi$ mesons, (middle) their invariant mass, and (right) the pair $p_{\mathrm{T}}$. Overlaid are the predictions from leading order DPS and NLO* SPS contributions. Figures are taken from Ref. [6].

\section{$5 X(3872)$ production measurement}

The $X(3872)$ particle was first discovered by the Belle experiment in 2003 and later confirmed by many others. Its mass, narrow width, quantum numbers and decay properties measured in several experiments make it unlikely to be a conventional quarkonium state. There is no final theoretical picture for this particle yet and various theoretical models are proposed in the literature to describe its structure.

ATLAS has studied the production of $X(3872)$ in the decay mode $X(3872) \rightarrow J / \psi \pi^{+} \pi^{-}$, using the decay $\psi(2 S) \rightarrow J / \psi \pi^{+} \pi^{-}$as a normalization channel [7]. The analysis is done with the $p p$ collision data collected at $\sqrt{s}=8 \mathrm{TeV}$ corresponding to an integrated luminosity of $11.4 \mathrm{fb}^{-1}$.

The production cross-section is measured in five bins of $p_{\mathrm{T}}$. In order to separate the prompt and non-prompt production, the data in each $p_{\mathrm{T}}$ bin are split into intervals of pseudoproper lifetime. An attempt to describe the non-prompt component of the signal distribution using a single effective lifetime, $\tau_{\text {eff }}$, results in smaller values of $\tau_{\text {eff }}$ at low $p_{\mathrm{T}}$ for $X(3872)$, while for the $\psi(2 \mathrm{~S})$ it is measured to be uniform across the $p_{\mathrm{T}}$ range. This possibly indicates a different $X(3872)$ production mechanism at low $p_{\mathrm{T}}$. An alternative model allows for two nonprompt contributions with different effective lifetimes, assuming the short-lived component to originate from the $B_{c}^{+}$meson decays. The fraction of this short-lived component in a range 
$p_{\mathrm{T}}(X(3872))>10 \mathrm{GeV}$ is measured to be

$$
\left.\left.\left.\frac{\sigma\left(p p \rightarrow B_{c}^{+}+\text {any }\right) \mathcal{B}\left(B_{c}^{+} \rightarrow X(3872)+\text { any }\right)}{\sigma(p p \rightarrow \text { non-prompt } X(3872)+\text { any })}=(25 \pm 13 \text { (stat. }) \pm 2 \text { (syst. }\right) \pm 5 \text { (spin }\right)\right) \% .
$$

Since the $B_{c}^{+}$production makes up only a small fraction of the inclusive beauty production, this value of the ratio would mean that the production of $X(3872)$ in $B_{c}^{+}$decays is strongly enhanced compared to its production in the decays of other $b$ hadrons.

Figure 8 shows the measured $X(3872)$ differential production cross-section for prompt and non-prompt production. The prompt production is well described by the predictions based on NLO NRQCD. The FONLL predictions of the non-prompt $\psi(2 \mathrm{~S})$ production, recalculated to $X(3872)$ using the branching fraction extracted from the Tevatron data, significantly overestimate the non-prompt production of $X(3872)$, especially at large transverse momenta.
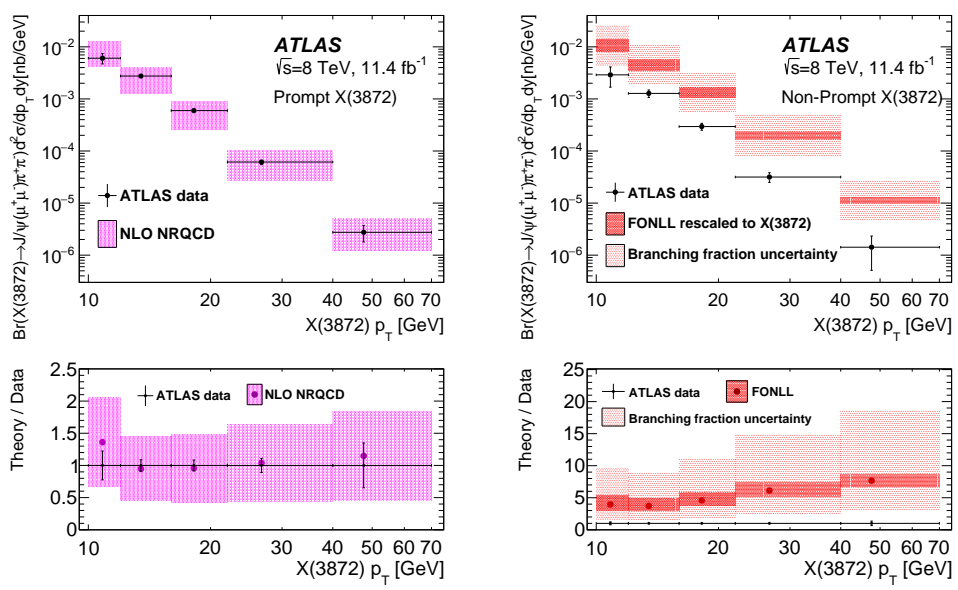

Figure 8. Measured cross-section times branching fraction as a function of $p_{\mathrm{T}}$ for (left) prompt and (right) non-prompt $X(3872)$ compared to theoretical expectations. Figures are taken from Ref. [7].

\section{Physics of $B_{c}$ mesons}

The states of the $B_{c}$ meson family are unique in the Standard Model, containing two different flavour heavy quarks. A number of calculations exist describing the spectroscopy of such system, as well as its production and decays. Although the ground state, the $B_{c}^{+}$meson, was discovered at Tevatron, only at the LHC it became possible to perform a wide range of studies of its characteristics and searches for excited states, providing an excellent test ground for the existing theoretical calculations.

First observation of an excited state of $B_{c}$ meson was reported by the ATLAS experiment [8]. The analysis used the full Run-1 $p p$ collision dataset collected at $\sqrt{s}=7$ and $8 \mathrm{TeV}$, corresponding to integrated luminosities of $4.9 \mathrm{fb}^{-1}$ and $19.2 \mathrm{fb}^{-1}$, respectively. The state was searched in a decay to the $B_{c}^{+}$meson and two charged pions with the $B_{c}^{+}$being reconstructed in the $B_{c}^{+} \rightarrow J / \psi \pi^{+}$decay. The signal was observed in a distribution of the mass difference $Q=m\left(B_{c}^{+} \pi^{+} \pi^{-}\right)-m\left(B_{c}^{+}\right)-2 m\left(\pi^{+}\right)$. Figure 9 shows this distribution for $7 \mathrm{TeV}$ and $8 \mathrm{TeV}$ data with clear signal peaks visible in both. The statistical significance of the signal in the combined dataset is 5.2 standard deviations. 

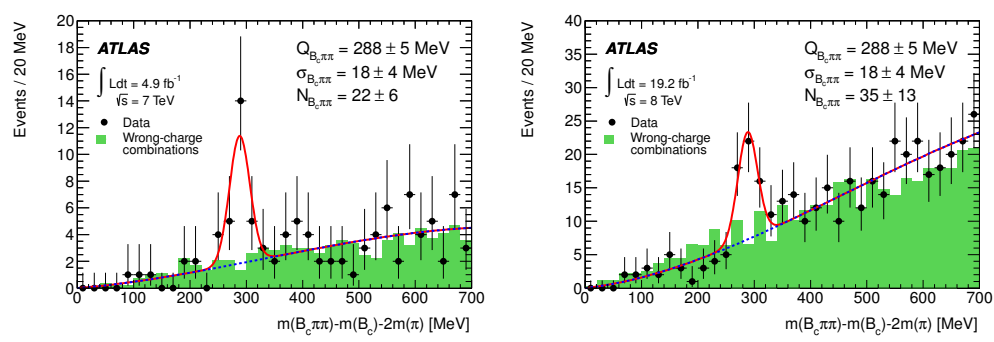

Figure 9. The $Q$ distribution for the right-charge combinations (points with error bars) and for the same (wrong) pion charge combinations (shaded histogram) in (left) $7 \mathrm{TeV}$ and (right) $8 \mathrm{TeV}$ data. Figures are taken from Ref. [8].

The mass corresponding to the observed signal, $6842 \pm 4$ (stat.) \pm 5 (syst.) $\mathrm{MeV}$, is consistent with the expectations for second $S$-wave excitations of the $\bar{b} c$ system, $B_{c}(2 S)^{+}$and $B_{c}^{*}(2 S)^{+}$. Thus the signal can be attributed either to the decay $B_{c}(2 S)^{+} \rightarrow B_{c}^{+} \pi^{+} \pi^{-}$or to the decay $B_{c}^{*}(2 \mathrm{~S})^{+} \rightarrow B_{c}^{*+} \pi^{+} \pi^{-}$with a subsequent transition $B_{c}^{*+} \rightarrow B_{c}^{+} \gamma$ where the photon is not reconstructed, or to a combination of the two processes.

Later the LHCb collaboration claimed [9] that no $B_{c}^{(*)}(2 S)^{+}$signals are seen in the same final states in the data collected during Run-1 and Run-2. Upper limits set on the relative $B_{c}^{(*)}(2 S)^{+}$production rate appear to be in a tension with the ATLAS result, although it is not possible to make a definite conclusion on the consistency. This tension clearly motivates further studies by the ATLAS and CMS experiments, in particular searches for these excitations in the Run-2 $p p$ collision data.

\section{Summary}

ATLAS experiment has a wide and solid programme in heavy flavour physics, largely including studies of charm sector. Some recent highlights were presented in this paper. Most of the results are obtained with the Run-1 data. Many studies suffer from limited available data statistics. Significantly larger available sample of $p p$ collision data collected during the Run2 , as well as upgrades of the detector and trigger system, will allow substantial improvements of their precision and sensitivity.

The work presented was partially supported by RFBR according to the research project 18-32-00825.

\section{References}

[1] ATLAS Collaboration, JINST 3, S08003 (2008)

[2] ATLAS Collaboration, Eur. Phys. J. C 76, 283 (2016), arXiv: 1512.03657 [hep-ex]

[3] ATLAS Collaboration, ATLAS-CONF-2015-030, https://cds.cern.ch/record/ 2037967

[4] ATLAS Collaboration, Eur. Phys. J. C 78, 762 (2018), arXiv: 1805.04077 [hep-ex]

[5] ATLAS Collaboration, Eur. Phys. J. C 78, 171 (2018), arXiv: 1709.03089 [hep-ex]

[6] ATLAS Collaboration, Eur. Phys. J. C 77, 76 (2017), arXiv: 1612.02950 [hep-ex]

[7] ATLAS Collaboration, JHEP 01, 117 (2017), arXiv: 1610.09303 [hep-ex]

[8] ATLAS Collaboration, Phys. Rev. Lett. 113, 212004 (2014), arXiv: 1407.1032 [hep-ex]

[9] LHCb Collaboration, JHEP 01, 138 (2018), arXiv: 1712 .04094 [hep-ex] 